\title{
Erratum to: Surgical robotics beyond enhanced dexterity \\ instrumentation: a survey of machine learning techniques and their role in intelligent and autonomous surgical actions
}

\author{
Yohannes Kassahun ${ }^{1}$ - Bingbin $\mathbf{Y u}^{2}$ - Abraham Temesgen Tibebu ${ }^{2}$. \\ Danail Stoyanov $^{3}$ - Stamatia Giannarou ${ }^{4}$ - Jan Hendrik Metzen ${ }^{2}$. \\ Emmanuel Vander Poorten ${ }^{5}$
}

Published online: 11 January 2016

(C) CARS 2016

\section{Erratum to: Int J CARS DOI 10.1007/s11548-015-1305-z}

The correct Table 1 is given here.

Unfortunately, the online published article has errors in Table 1. The line after the first row of Table 1 should be removed.

Table 1 Aspects of autonomous robotic surgery (ARS) where ML could play an enabling role

\begin{tabular}{ll}
\hline Workflow analysis episode segmentation & $\begin{array}{c}\text { Surgical procedure broken down into logical subtasks or episodes } \\
\text { Environment modeling }\end{array}$ \\
Rigid and flexible registration, reconstruction of environment, recognition of anatomical \\
features and landmarks, mechanical and physiological modeling \\
Localization & $\begin{array}{l}\text { Localization of instrument/robot w.r.t. environment } \\
\text { Lobot control }\end{array}$ \\
Skill analysis & $\begin{array}{l}\text { Analysis of surgical skill, derivation of performance metrics or cost functions for } \\
\text { optimization }\end{array}$ \\
Critical event detection & Detection of adverse events \\
Planning and control & High-level trajectory and interaction planning, error handling \\
\hline
\end{tabular}

The online version of the original article can be found under doi:10.1007/s11548-015-1305-z.

Yohannes Kassahun

yohannes.kassahun@dfki.de

1 Robotics Innovation Center, German Research Center for Artificial Intelligence, Robert-Hooke-Str. 1, 28359 Bremen, Germany

2 Faculty 3 - Mathematics and Computer Science, University of Bremen, Robert-Hooke-Str. 1, 28359 Bremen, Germany

3 Centre for Medical Image Computing, Department of Computer Science, University College London, London, UK

4 Hamlyn Centre for Robotic Surgery, Imperial College London, London, UK

5 Department of Mechanical Engineering, University of Leuven, Celestijnenlaan 300B, 3001 Heverlee, Belgium 